\section{DIÁNOIA}

DE DONATO, XAVIER

El carácter de los tipos ideales weberianos y su relación con las ciencias naturales

Diánoia, vol. LII, núm. 59, noviembre, 2007, pp. 151-177

Universidad Nacional Autónoma de México

Distrito Federal, México

Disponible en: http://www.redalyc.org/articulo.oa?id=58433531007

- Cómo citar el artículo

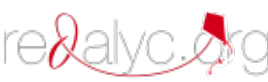

- Número completo

- Más información del artículo

- Página de la revista en redalyc.org

Sistema de Información Científica

Red de Revistas Científicas de América Latina, el Caribe, España y Portugal

Proyecto académico sin fines de lucro, desarrollado bajo la iniciativa de acceso abierto 


\title{
El carácter de los tipos ideales weberianos y su relación con las ciencias naturales*
}

\author{
XAVIER DE DONATO \\ Instituto de Investigaciones Filosóficas \\ Universidad Nacional Autónoma de México \\ xdonato@minerva.filosoficas.unam.mx
}

\begin{abstract}
Resumen: En este artículo examino la relación entre los conceptos ideales de las ciencias naturales y los tipos ideales propuestos por Max Weber como parte específica de la metodología en ciencias humanas. Señalaré algunas dificultades de los análisis realizados hasta el momento en este sentido. Mi tesis principal es que en la noción de tipo ideal defendida por Weber (1921) sí se encuentra un elemento decisivo que haría posible establecer una similitud importante con el estatus de los conceptos ideales en ciencias naturales. A pesar de ello, las consecuencias que se derivan de esta comparación no son suficientes para negar la diferencia metodológica que Weber vio entre ambos tipos de ciencia.
\end{abstract}

Palabras clave: conceptos ideales, idealización, metodología de Max Weber, demarcación entre ciencias naturales y sociales

\begin{abstract}
In the present paper, I examine the relation between the ideal concepts of natural sciences and the ideal types proposed by Max Weber as a specific part of the methodology in human sciences. I will present some difficulties in the accounts which have been proposed so far in this sense. My main thesis will be that in Weber's notion of "ideal type" there is indeed an element which makes it possible to establish an important comparison with the ideal concepts in natural sciences. Notwithstanding, the consequences drawn from this comparison are not sufficient for neglecting the methodological difference Weber ascribed to both kinds of sciences.

Key words: ideal concepts, idealization, Max Weber's methodology, demarcation between natural and social sciences
\end{abstract}

\section{Introducción}

Uno de los primeros filósofos en señalar la importancia de la idealización en la ciencia como un componente esencial de las teorías científicas y, en especial, en la construcción de las nociones teóricas, y en tratarla de un modo explícito en el marco de una discusión metodológica me parece que fue Max Weber. Puesto que los intereses de Weber siempre se centraron en las ciencias sociales y no en las naturales, y

*Agradezco al doctor Raymundo Morado sus comentarios a una versión previa de este artículo, así como a los árbitros de Diánoia.

Diánoia, volumen LII, número 59 (noviembre 2007): pp. 151-177. 
puesto que su concepción de los tipos ideales se enmarca en un planteamiento epistemológico que parte de la distinción entre ambas familias de ciencias, la comunidad de filósofos de la ciencia no específicamente orientados a las ciencias sociales ha tendido a dejar de lado o simplemente a ignorar las ideas metodológicas del autor alemán. Excepciones a esta situación general han sido, por ejemplo, Hempel (1965), Papineau (1976), Jeffry L. Ramsey (1994), Ibarra y Mormann (1998) y, más recientemente, Camardi (2004). Todos estos artículos intentan situar los planteamientos de Weber en el marco de la discusión general sobre explicación e idealización en ciencias naturales. En el presente artículo pretendo seguir sus pasos y estudiar la relación entre los tipos ideales weberianos y los conceptos ideales con que se trabaja habitualmente en ciencias naturales. Sin embargo, mi análisis y mis conclusiones van a separarse en algún punto esencial de mis predecesores:

(1) contra Hempel y Papineau, argumentaré que ni los tipos ideales en ciencias sociales ni los conceptos ideales en ciencias naturales necesitan la existencia de una teoría científica que les sirva de cobertura;

(2) contra Papineau en particular, sostendré que las idealizaciones sí pueden ser testadas empíricamente;

(3) contra Ramsey, mantendré que tanto en las ciencias naturales como en las humanas los conceptos y tipos ideales son difícilmente instanciables si es que alguna vez lo son, aunque ambos tipos de ciencia van a diferir en el modo y posibilidad de esa no instanciación. El problema va ser cómo dar cuenta de (2) a pesar de (3);

(4) finalmente, a pesar de estar de acuerdo con Ibarra y Mormann, así como con Camardi, en la oportunidad de señalar la asimilabilidad de los tipos ideales a los conceptos ideales de las ciencias naturales, mostraremos que esa asimilación puede realizarse sólo con ciertas salvedades, o mejor: es posible e incluso adecuado hacerla en el caso de los tipos ideales concebidos tal y como lo hace Weber en su obra tardía, especialmente en su obra magna publicada póstumamente, pero inadecuada si tenemos en cuenta la concepción de los tipos ideales formulada en 1904.

Como la literatura especializada lo ha notado, ${ }^{1}$ ese cambio en la concepción de los tipos ideales se opera a partir de "Über einige Kategorien

${ }^{1}$ Véase, por ejemplo, Mommsen 1989, p. 127, y también Aguilar Villanueva 1989, pp. 638 y ss.

Diánoia, vol. LII, no. 59 (noviembre 2007). 
der verstehenden Soziologie", de Weber (1913). Esta doble concepción, que no es mutuamente excluyente, ni siquiera exhaustiva, no ha sido debidamente atendida a la hora de hacer un análisis comparativo con las ciencias naturales. Por otro lado, asimilando sin ulteriores distinciones los tipos ideales weberianos a los conceptos ideales de las ciencias naturales, se corre el riesgo de asimilar, a nivel conceptual y explicativo, las ciencias humanas a las naturales y contradecir así la principal motivación de Weber al introducir los tipos ideales: trazar una clara línea divisoria, a nivel especialmente metodológico, entre ambos tipos de ciencias. Independientemente de lo que uno piense acerca del estatus de las ciencias humanas y de su relación con las ciencias naturales, una cuestión importante es examinar qué luz arroja la comparación entre tipos ideales y conceptos ideales en ciencias naturales en relación con el problema de la demarcación entre ciencias humanas y ciencias naturales. Resultaría un tanto extraño valerse de un concepto que fue introducido para fundamentar esa división justo para lograr lo contrario; es decir, anularla. Sin embargo, muchos de los trabajos realizados hasta ahora sobre la relevancia de los tipos ideales para las ciencias naturales, como los de Ramsey, Ibarra y Mormann o Camardi, no tienen en cuenta esta cuestión y parecen ir en contra de la idea general de Weber de una separación entre ambos tipos de ciencia.

Por tanto, un primer nivel de refinamiento que pretendo haber logrado en mi comparación entre tipos weberianos y conceptos ideales de las ciencias naturales consiste en haber considerado la distinción entre las dos nociones de tipo ideal y su relevancia para la comparación en cuestión. El segundo nivel de refinamiento es no prejuzgar la cuestión de la demarcación entre ambos tipos de ciencia y analizar la relevancia de nuestra comparación para el problema de la demarcación.

Hemos hablado de dos nociones de tipo ideal. La primera acepción corresponde a los llamados "tipos ideales individuales", que, como veremos, tienen la función de procurar el conocimiento y la comprensión de los fenómenos individuales o sociales de carácter singular. Esta primera concepción está vinculada a una primera etapa en el pensamiento metodológico weberiano dependiente básicamente de la historia (sin ser historicista). La segunda acepción corresponde a los tipos ideales generales de la sociología, disciplina en la que Weber se centró durante una segunda etapa de su obra. No es que Weber rechazara la primera noción de tipo ideal en favor de la segunda: ambas nociones de tipo ideal son usadas legítimamente en las ciencias humanas, o culturales para emplear el término de Weber, sólo que la segunda va a tener una importancia capital en los fundamentos metodológicos de la sociolo- 
gía. Tanto la primera como la segunda caracterización que hace Weber de los tipos ideales son, sin embargo, insuficientes y están formuladas en términos poco precisos y hasta difícilmente compatibles, lo que ha llevado a confusiones e interpretaciones opuestas. ${ }^{2}$ Las dos nociones mismas, como veremos, se han llegado a confundir entre sí o no se ha reparado lo suficiente en su distinción, lo que ha ocasionado críticas, a mi entender, injustificadas al pensamiento weberiano como la de von Mises (1929). Si bien la crítica de este último se puede aplicar con fundamento a la primera noción de tipo ideal, no así a la segunda, según puso de manifiesto tempranamente Alfred Schütz (1993, p. 270).

La estructura de este trabajo es como sigue. En la sección 2 nos referiremos brevemente a la naturaleza y función metodológica de los tipos ideales weberianos: su doble caracterización por parte de Weber. En la sección 3 estudiaremos la relación entre los tipos ideales weberianos y los conceptos ideales en ciencias naturales. Discutiremos específicamente las contribuciones de Watkins (1952), Brown (1963), Hempel (1965) y Nowak (1980). En la sección 4, nos centraremos exclusivamente en los conceptos ideales de las ciencias físicas, pues un estudio de ellos en las ciencias naturales en general complicaría y alargaría en exceso este trabajo. La relevancia de los tipos ideales para otras ciencias naturales que, como las ciencias históricas y sociales, también persiguen explicaciones de hechos singulares, como la astronomía, la geología o la arqueología, queda fuera de nuestra discusión, pero puede postergarse con vistas a un artículo futuro.

\section{Naturaleza y función de los tipos ideales weberianos}

Según Weber, un "tipo ideal" ("Idealtypus", en alemán) es una construcción mental (o "Gedankenbild") que tiene "el carácter de una utopía en sí, que es obtenida a partir de la exageración mental de determinados elementos de la realidad". ${ }^{3}$ Tal construcción no debe implicar una contradicción lógica, pero "no corresponde al mundo real". Podemos interpretar esto diciendo que un tipo ideal es un concepto o representación mental (situado en la mente del historiador, del sociólogo o del teórico económico) cuya descripción describe un estado de hechos lógicamente posible, pero que es difícil encontrar en el mundo real. Weber

${ }^{2}$ Von Schelting (1934, p. 332) y otros especialistas han repararado en la equivocidad del concepto.

${ }^{3}$ Weber 1904, p. 42; la traducción es mía. El original dice: "den Character einer Utopie an sich, die durch gedankliche Steigerung bestimmter Elemente der Wirklichkeit gewonnen ist".

Diánoia, vol. LII, no. 59 (noviembre 2007). 
está pensando en cierto tipo de constructos conceptuales en ciencias humanas y, más específicamente, en ejemplos del tipo cultura capitalista o economía de estado medieval. Nociones como mercado perfecto u hombre económico son también ejemplos bien conocidos de tipos ideales en teoría económica.

Según Max Weber, el uso de estos conceptos es peculiar de las ciencias humanas frente a las ciencias naturales. De acuerdo con Weber (1904), el científico social no debe proceder como en las ciencias naturales; a saber, intentando sistemáticamente subsumir enunciados de observación bajo leyes generales, cuyo descubrimiento sería la finalidad de la ciencia, sino que debe dar explicaciones de eventos particulares a través de hipótesis que refieran a la relación causal entre estos eventos y otros eventos particulares, porque es en el fenómeno particular en su singularidad, el evento-particular y no el evento-tipo, en lo que está interesado el científico social; es decir, su objetivo no es establecer leyes generales y entonces subsumir el fenómeno que se va a explicar como caso particular, sino explicar el fenómeno qua particular. Ejemplos de eventos particulares que le interesan al historiador o al teórico económico son, por ejemplo, "la derrota de Napoleón en Waterloo" o "la caída de la bolsa de Nueva York en 1929". El uso de tipos ideales debe ayudar al científico social en su intento de explicación de estos fenómenos.

Esta idea de la demarcación entre ciencias naturales e históricas o sociales ("culturales", dice también Weber) no es algo específico de Weber, sino que éste lo toma de Windelband y Rickert, dos filósofos alemanes de la escuela de Baden que influyeron decisivamente en la epistemología y la metodología weberianas. ${ }^{4}$ También fueron la no recurrencia a leyes generales y la referencia a relaciones causales entre particulares dos aspectos decisivos en la crítica al modelo de cobertura legal de tipo hempeliano que autores como von Wright o Dray realizaron en su análisis de la explicación en historia y ciencias sociales.

Si los tipos ideales resultan de tanta ayuda en ciencias sociales, cualquier información acerca del estatus lógico de estos conceptos y la manera en que típicamente los construimos debe interesar a los metodólogos; pero, desafortunadamente, Weber (1904) es aquí muy impreciso. De acuerdo con él, los tipos ideales no son hipótesis en sí mismos, pero pueden tener una función heurística y ayudar a construir hipótesis. No son conceptos de clase, sino conceptos límite formados

${ }^{4}$ Véase, por ejemplo, Rickert 1986, pp. 96 y ss. Para un análisis en español de las ideas de Weber a este respecto y su comparación con las de Windelband o Rickert, véase por ejemplo Aguilar Villanueva 1988 y 1989, 2 vols., especialmente el vol. 2, pp. 494 y ss. 
por síntesis ("Zusammenschluß" o "abstrahierende Zusammenfassung" son los términos que utiliza) de varios y difusos eventos individuales concretos "más o menos presentes y ocasionalmente ausentes", que son organizados, de acuerdo con ciertos puntos de vista, ${ }^{5}$ para formar un constructo conceptual unificado ("in sich einheitlichen Gedankenbilde" (Weber 1904, p. 191)). El juicio de Weber acerca del estatus lógico de los tipos ideales es, pues, fundamentalmente negativo; pero

cuando se trata de una caracterización positiva, recurre a un lenguaje muchos menos preciso que con frecuencia es metafórico [...]. Esta caracterización y muchos planteos similares que Weber y otros han hecho acerca de la naturaleza de los tipos ideales son ciertamente sugestivos, pero les falta claridad y rigor, por lo cual requieren un análisis lógico más profundo. (Hempel 1965, p. 160)

De ahí la necesidad de emprender un análisis de los tipos ideales como el de Hempel o, como mínimo, de comparar su función en ciencias sociales con la de los conceptos ideales habitualmente empleados en ciencias naturales.

El uso de tipos ideales hace posible comprender fenómenos sociales o históricos concretos a través de la comparación entre el tipo ideal y el fenómeno concreto. Weber otorga a sus tipos ideales una función heurística en un doble nivel, en el de la exposición y en el de la investigación. En el primero, nos sirven para tejer un discurso conceptual y, en el segundo, para construir a partir de ellos hipótesis explicativas de tipo individual (Aguilar Villanueva 1989, vol. 2, pp. 570-571). Hemos visto que ejemplos de tipos ideales son cultura capitalista, economía artesanal o economía de estado medieval. Se trata, hemos dicho, de conceptos límite que no son realizados de forma exacta o completa en el mundo real. Podemos preguntarnos si en este sentido no se parecen a los conceptos ideales usados en ciencias naturales, tales como cuerpo rígido, gas ideal o cuerpo perfectamente elástico. De hecho, sí son similares: estos conceptos son justamente ideales porque no se hallan ejemplificados en la realidad sino sólo aproximadamente, pertenecen a teorías científicas y son construidos con el fin de hacer explicaciones. La principal diferencia que puede ofrecerse primeramente es el hecho de que los tipos ideales weberianos son constructos válidos únicamente para explicar eventos particulares y no para explicar un conjunto de eventos particulares, no para explicar eventos tipo. Los ejemplos de tipos ideales en física que

\footnotetext{
${ }^{5}$ Por tanto, es de suponerse que disitintos puntos de vista o perspectivas conducirán a tipos ideales diferentes.
}

Diánoia, vol. LII, no. 59 (noviembre 2007). 
acabamos de mencionar son aplicados a conjuntos de objetos particulares; es decir, a tipos de objetos acerca de los cuales son verdaderas o se pretenden verdaderas ciertas leyes.

Hagamos notar, antes de abandonar este apartado, que tratándose como se trata en este artículo no tanto de la discusión de los tipos ideales en ciencias sociales, cuanto de una comparación de la metodología weberiana con la de las ciencias naturales, habremos de omitir otras problemáticas muy importantes en historia o sociología, como son las cuestiones de la comprensión de sentido o la "doble hermenéutica" (en el sentido de Giddens y Habermas). Un examen detenido de la función de los tipos ideales en la explicación en ciencias humanas, así como de su construcción misma como conceptos, exigiría lidiar con estos problemas. ${ }^{6}$ El modesto objetivo del presente artículo nos evita, sin embargo, tener que hacerlo aquí; de esta forma dejo este punto tan importante para una posterior ocasión.

\section{La relación de los tipos ideales con las ciencias naturales}

Hasta aquí una primera diferencia entre tipos ideales weberianos y los conceptos ideales empleados habitualmente en ciencias naturales. Veamos algunas trayendo a colación dos nociones más habitualmente discutidas en filosofía de la ciencia: las de tipo extremo e idealización. Brown (1963) y Hempel (1965) están entre los autores que han distinguido entre tipos extremos e idealizaciones. Para ambos, "tipos extremos" son puntos límite de una serie ordenada bajo ciertos criterios. Pero mientras Hempel identifica los tipos ideales weberianos con idealizaciones, para Brown algunos de los tipos ideales weberianos son tipos extremos y no idealizaciones; por ejemplo: "sociedad popular pura", "secta protestante" o "comunidad" (en el sentido técnico de "Gemeinschaft"), resultando la diferencia más relevante que las idealizaciones son asunciones de condiciones "físicamente imposibles" (según Brown), mientras que éste no sería el caso de los tipos extremos: una sociedad puramente comunista seguramente no existe hoy día, pero podría existir en el sentido de que es físicamente realizable (Brown 1963, pp. 179180). De acuerdo con Brown, las idealizaciones son extrapolaciones de un valor límite de ciertas variables: en el caso de los gases ideales, por ejemplo, los volúmenes y las masas de las moléculas asumen el valor 0; en los cuerpos perfectamente rígidos, es decir, en aquellos en que la

${ }^{6}$ Estas ideas ya están presentes, por cierto, en las reflexiones de Weber (1904, y 1921) sobre sus tipos ideales. 
distancia entre cualesquiera dos puntos se mantiene invariable, si una fuerza es aplicada sólo en un punto, entonces esa fuerza debe ser transmitida instantáneamente a todos los otros puntos del cuerpo, lo que es tanto como decir que esa fuerza es transmitida con una velocidad infinita. La realización de estas condiciones ideales es físicamente imposible: desde el punto de vista de la teoría especial de la relatividad, energía y momento, y por tanto, la fuerza, no pueden ser transmitidos con una velocidad mayor que la de la luz. No obstante, el concepto de cuerpo rígido es empleado en la física relativista. Parece también físicamente imposible la realización del concepto ideal de punto masa (con volumen cero, como en el caso de las moléculas de los gases ideales), pues el potencial gravitacional infinito que resultaría de concentrar la masa gravitacional en un punto sin dimensiones contradice las leyes de la mecánica clásica. Esta distinción entre tipos extremos e idealizaciones, tal y como la realiza Brown, recuerda la distinción hecha por Suppe (1989, pp. 94-96) entre abstracción (que refiere a condiciones que son causal o nomológicamente posibles) e idealizaciones (que refieren a condiciones que no son causal o nomológicamente posibles).

Un ejemplo paradigmático de idealización (en este sentido) es el de hombre económico. Al igual que ocurre con el de gas ideal, el concepto hombre económico involucra ciertas asunciones ideales hechas por los economistas para caracterizar el concepto, asunciones tales como la completa racionalidad del hombre económico o la perfecta movilidad en todos los ajustes económicos. Sobre estas asunciones, Brown dice: "Es obvio que cada una [de ellas] es insatisfactible —es físicamente imposible- en nuestro mundo." ${ }^{7}$ Podemos aceptar que gran parte de esas asunciones sean difíciles de ser satisfechas en la realidad de acuerdo con las leyes de la psicología (como la asunción de perfecta racionalidad y entera independencia de acción en relación con las otras personas y respecto de los prejuicios sociales) o incluso de la economía (como la asunción de que no hay otro medio de adquirir bienes aparte de la producción y el libre cambio en el mercado abierto, asunciones que ignoran conscientemente la existencia de un mercado negro, cuya influencia en el mercado puede ser explicada por teorías económicas avanzadas), pero estas asunciones no van obviamente contra leyes físicas; luego, el uso de "físicamente posible" que hace Brown es desafortunado. Caritativamente, hay que entender que Brown se está refiriendo no a asunciones que van contra las leyes de la física, sino contra los

${ }^{7}$ Brown 1963, p. 181; la traducción es mía. 
hechos establecidos en la economía o la psicología, exactamente como sucede en la física con conceptos ideales como gas ideal, que violan, estrictamente hablando, ciertas leyes de la física. Pero incluso esta interpretación de los tipos ideales económicos o sociológicos es controvertida, pues en los casos de las ciencias sociales hablar de que ciertas asunciones contradicen ciertas leyes sociales o psicológicas puede resultar como mínimo discutible. Así por ejemplo, un teórico económico como Frank Knight, que desarrolla una teoría particular del hombre económico y del mercado perfecto, considera que sus asunciones que no difieren de las de Brown-son "idealizaciones o purificaciones de tendencias que son satisfechas más o menos en la realidad", 8 desde luego no de modo exacto, pero en cualquier caso son de realización perfectamente posible. Así, como descripciones de estados de hechos no realizados pero empíricamente posibles, interpreta Burger (1987) los tipos ideales weberianos. ${ }^{9}$

Como Brown, Hempel (1965) distingue entre "tipos extremos" e "idealizaciones"; pero, según Hempel, los tipos ideales weberianos corresponden sólo a las últimas, no a los primeros. Ejemplos de tipos extremos en el sentido hempeliano son conceptos psicológicos como "personalidades puramente extrovertidas" o "puramente introvertidas", los cuales son raramente (si es que alguna vez) instanciados, pero que pueden servir como puntos límite de una serie, como en el caso de la caracterización de Brown. Según Hempel, uno no construye este tipo de conceptos como conceptos de clase, sino a partir de una relación comparativa: para cualesquiera dos casos en el universo de investigación $a$ y $b$, basta con tener criterios para establecer si $a$ es más $C$ que $b$ o es $\tan C$ como $b$, donde $C$ es el concepto comparativo en cuestión. Los tipos ideales weberianos son concebidos por Hempel más bien como idealizaciones y, por tanto, su estatus sería, según él, diferente del de los tipos extremos. De acuerdo con Hempel, los tipos ideales usados, por ejemplo, en teoría económica funcionarían como idealizaciones en las ciencias naturales; no habría, pues, diferencia como pretende Weber entre unas ciencias y otras. Existen, sin embargo, según él, dos importantes diferencias: las idealizaciones en teoría económica son más intuitivas que teóricas en el sentido de que los postulados o hipótesis que corresponden a estas idealizaciones no son deducidos

\footnotetext{
${ }^{8}$ Knight 1921, p. 79; la traducción y las cursivas son mías.

${ }^{9}$ Los tipos ideales "describen fenómenos que en principio son empíricamente posibles pero que no han conseguido materializarse como un resultado de circunstancias factuales" (Burger 1987, p. 124; la traducción es mía).
} 
de una teoría más amplia que cubra también los factores no racionales y no económicos que afectan la conducta humana. No existe por el momento una teoría adecuada más general y por lo tanto no hay base teórica para una apreciación de la idealización que se maneja al aplicar las construcciones económicas a situaciones concretas. (Hempel 1965, p. 173)

Por otro lado, y ésta es la segunda diferencia, la clase de fenómenos conductuales concretos que el economista trata de idealizar (es decir, su dominio de aplicación) no siempre está claramente especificada. Para Hempel, la situación es incluso peor en el caso de los tipos ideales empleados en ciencias sociales diferentes de la economía. Sin embargo, a pesar de estas diferencias, Hempel concibe los tipos ideales weberianos no como algo peculiar de las ciencias sociales, sino como algo que tiene sustancialmente el mismo carácter que las idealizaciones de las ciencias naturales, las cuales no tienen el estatus de conceptos en sentido restringido, sino más bien de sistemas teóricos. La explicación en las ciencias sociales se vuelve así algo no distinto de la explicación en ciencias naturales.

Como a continuación voy a argüir, los ejemplos, citas y explicaciones hechas por Hempel de la concepción weberiana de los tipos ideales o bien no tiene en cuenta dos diferentes caracterizaciones de tipo ideal en Weber, o bien las asimila conscientemente. Esta diferencia, sin embargo, existe y ha sido notada por autores como Watkins (1952), que habla de "tipos ideales holísticos", por un lado, y de "tipos ideales individualísticos", por el otro. Los primeros corresponden a lo que Weber llama tipo ideal en su estudio sobre la objetividad en las ciencias sociales (1904); los segundos corresponden a la caracterización que Weber hace de los tipos ideales en el primer capítulo de su póstumo Wirtschaft und Gesellschaft (1922). Tipos ideales en el primer sentido son tales como los ya descritos, es decir, conceptos abstractos, difíciles de encontrar ejemplificados en la realidad, que el teórico construye con el fin de explicar ciertos fenómenos sociales particulares. Se trata más bien de "tipos extremos" (en el sentido que esta expresión tiene según hemos visto para Brown) que de idealizaciones; ejemplos de ellos son "cultura capitalista", "industria artesanal" o "secta protestante". Tipos ideales en el segundo sentido son más bien idealizaciones en el sentido de Hempel y tienen un sentido contrafáctico explícitamente advertido por Weber (1921), siendo un ejemplo paradigmático de ellos el de "hombre económico".

Nowak (1980) también parece ignorar esta diferencia; de hecho, cuando cita a Weber sólo lo hace a partir de su trabajo de 1904. El tema 
central del libro de Nowak es la idea de la ciencia de Karl Marx y el método idealizacional seguido por Marx en El capital. Nowak ve en Marx al "Galileo de las ciencias sociales": así como Galileo reaccionó frente a la física aristotélica, basada en el sentido común y la pura observación, e introdujo el método de la idealización en la física, Marx reaccionó contra la economía vulgar, que prestó atención únicamente a la apariencia de los fenómenos, e introdujo el método de la idealización en la economía, intentando revelar la verdadera naturaleza de los fenómenos económicos y las estructuras del sistema económico burgués. Marx mismo llamó a su método "abstracción" —utiliza esta misma expresión en la primera página de El capital-; de acuerdo con este método, el economista "debe separar los factores principales de los secundarios en relación con un fenómeno dado y abstraer a partir de ellos estableciendo el fenómeno con sus principales determinantes". ${ }^{10}$ Nowak interpreta la abstracción de Marx como el método de la idealización y la concretización, reconstruyendo de acuerdo con su propio análisis algunas leyes de El capital, tales como la ley del valor. Al contrario que en el caso de Marx, Nowak no interpreta el método weberiano como el de la idealización y, puesto que según Weber todas las leyes marxianas y constructos teóricos son tipos ideales ${ }^{11}$ intenta mostrar que la convicción de Weber es falsa; es decir, que Marx no usó tipos ideales weberianos, sino el método de la abstracción (o idealización, para emplear el término de Nowak). ${ }^{12}$

El hecho, sin embargo, es que Weber (1921) se refiere explícitamente al carácter contrafáctico de la idealización, que aquí involucra nuevamente una relación causal entre eventos particulares: los conceptos y leyes de la economía teórica "representan cómo un determinado curso de acción humana tendría lugar, si estuviese orientado por un fin económico estrictamente racional no distorsionado por creencias erróneas ni emociones". ${ }^{13}$ Todos los errores e influencias emocionales son vistos como "Störungen" (factores interferentes). Al mismo tiempo, todas estas desviaciones irracionales de la acción humana son susceptibles de estudio desde un punto de vista sociológico y psicológico. Como se ha dicho, los tipos ideales weberianos deben ayudar a elaborar ex-

${ }^{10}$ Nowak 1980, p. 37; la traducción es mía.

${ }^{11}$ Véase Weber 1904, pp. 204-205.

${ }^{12}$ Véase Nowak 1980, cap. 3.

${ }^{13}$ Weber 1904, p. 548; la traducción es mía. El texto original dice: "stellen dar, wie ein bestimmt geartetes, menschliches Handeln ablaufen würde, wenn es streng zweckrational, durch Irrtum und Affekte ungestört, und wenn es ferner ganz eindeutig nur an einem Zweck (Wirtschaft) orientiert wäre". 
plicaciones causales hipotéticas. Para corroborar la validez de las leyes idealizadas, hemos de comparar nuestras hipótesis con el resultado real de la acción. Weber (1921) ve aquí dos posibilidades: o la comparación puede ser hecha con cierta precisión, porque involucra cuantificación (Weber está pensando aquí en explicaciones de tipo estadístico), o la comparación tiene lugar a través del método de aislamiento de factores causales: Weber habla explícitamente sobre la "eliminación hipotética de elementos individuales de la cadena de razones" (Weber 1921, p. 549). Es así como el procedimiento de Weber se asemeja al método de Nowak (y en general al de su escuela, la llamada escuela de Poznan), que estriba en la noción de negligencia de factores; es decir, en ignorar conscientemente la influencia de ciertos parámetros. Sin embargo, también es cierto que este método es sólo intuitivamente descrito por Weber (nunca explícitamente definido y, en particular, sin referencia explícita al proceso inverso a la idealización, la concretización).

De esto podemos concluir que el análisis de Nowak de la concepción de Weber no es correcto, justamente por no contemplar esta segunda noción de tipo ideal. De acuerdo con Nowak, los enunciados que refieren a tipos ideales no son sintéticos, sino analíticos y, según él, no pueden ser corroborados de ningún modo. Watkins (1952) parece ir en la misma dirección cuando dice de los tipos ideales holísticos (que son los tipos ideales tal y como los entiende Weber en su trabajo de 1904) que son definiciones, más concretamente definiciones estipulativas $\mathrm{y}$, por tanto, enunciados analíticos. Lo que Weber descarta al decir que no se trata de conceptos construidos a partir de un género y diferencia específica es que sean definiciones reales. Pero las definiciones pueden ser nominales o reales, ${ }^{14}$ y quizá los tipos ideales weberianos son del primer tipo. En todo caso, Hempel está esencialmente en lo correcto cuando dice que tipos ideales como cultura capitalista o economía artesanal representan teorías más que conceptos; esto es, teorías particulares sobre tales fenómenos económico-sociales (Weber habla de "perspectivas" en lugar de teorías). En principio, esto parece no concordar demasiado bien con la aserción de Weber de que los tipos ideales no son hipótesis; pero lo importante aquí no es que los tipos ideales sean identificables con hipótesis: lo que Hempel está diciendo es que los tipos ideales sólo cobran sentido cuando se los sitúa en el contexto de una teoría, donde estos conceptos están asociados a ciertos factores o parámetros y a un conjunto de hipótesis que conecta estos factores o parámetros entre sí (Hempel 1965, pp. 171-172).

\footnotetext{
${ }^{14}$ Véase Hempel 1965, cap. 1.
} 
Estas observaciones son válidas de los tipos ideales tal y como los concibe Weber en 1904. En relación con su posterior concepción, la de 1921, los tipos ideales no son ni definiciones ni enunciados analíticos, sino antecedentes de enunciados contrafácticos (o abreviaciones de tales antecedentes). No puedo estar de acuerdo, por tanto, con las premisas de Nowak de acuerdo con las cuales los enunciados que refieren a conceptos ideales son (i) analíticos, y (ii) pueden pero no necesariamente tienen que "desviarse de la realidad".

La premisa (ii) puede ser verdadera de los tipos ideales en el primer sentido, pero en ningún caso en el segundo sentido, porque en este segundo caso tienen, como hemos visto, un carácter contrafáctico. Sin embargo, Nowak puede estar en lo cierto cuando habla de que hay dos diferencias metodológicas entre las idealizaciones de Marx y los tipos ideales de Weber: en el trabajo de 1904, los tipos ideales tienen más una función clasificatoria, mientras que en el de 1921, pueden ser interpretados como tipos que tienen una función explicativa, como Hempel hace. Finalmente, la aserción de Nowak según la cual no hay lugar para la concretización en el modelo weberiano suena excesivamente radical (Nowak 1980, p. 50). Es verdad que no existe (yo por lo menos desconozco su existencia) un párrafo que explícitamente mencione un procedimiento de concretización o algo siquiera similar, pero eso no significa obviamente que el método de Weber no sea compatible con él. ${ }^{15}$

Para finalizar estas consideraciones sobre Weber, es necesaria una reflexión acerca del uso y función de ambas nociones de tipo ideal en la metodología weberiana. Algunos intérpretes de Weber han visto una suerte de incompatibilidad, rechazo o confusión en relación con estas dos nociones. Así, por ejemplo, Watkins (1952, p. 27) afirma que Weber rechazó la primera noción en favor de la segunda, y el teórico económico Walter Eucken llega incluso a criticar a Weber por confundir (según él) ambas nociones (Watkins 1952, p. 27). Según yo, sin embargo, Weber no rechazó su primera concepción de los tipos ideales para sustituirla por la segunda; para él, la primera caracterización siguió representando siempre una noción posible de tipo ideal, muy útil en ciencias sociales, junto con la otra caracterización, la contrafáctica, a la que presta mucha más atención en el estudio de 1921. De hecho, Weber (1921) vuelve incluso a su anterior caracterización al mencionar

${ }^{15}$ De hecho, los ejemplos de Weber sugieren el procedimiento de aislamiento de factores distorsionadores vía experimentos mentales, que podemos ver como una instanciación del método idealizacional. Véase más adelante y, además, Weber 1921, p. 561. 
ejemplos tales como "feudal" o "hereditario" y al decir que pueden ser aplicados al mismo fenómeno de acuerdo con diferentes perspectivas. Para dar sentido a estos conceptos, el científico social debe recurrir a tipos ideales, que define nuevamente como conceptos límite no contradictorios que son formados por síntesis y son difíciles de hallar ejemplificados en el mundo real. ${ }^{16}$

\section{Los conceptos ideales en ciencias naturales}

Por lo común, las idealizaciones en las ciencias naturales, y en particular en la física, ${ }^{17}$ son pensadas como conceptos o términos al igual que como enunciados; pero la tendencia por parte de ciertos autores a analizar la idealización de un modo sintácticamente unitario ha dado lugar a la discusión de si las idealizaciones (o en este contexto: los tipos ideales) son enunciados más bien que conceptos o términos. Rudner (1966), por ejemplo, ve esta ambigüedad y arguye que los conceptos ideales de la física como "superficie sin fricción" o "gas ideal" pueden ser definidos en términos de ciertas expresiones sentenciales (esto es, enunciados idealizacionales) y, por tanto, ser eliminables en favor de ellos; pero al mismo tiempo reconoce que no hay manera de distinguir desde un punto de vista puramente sintáctico entre una idealización y una no idealización (véase Rudner 1966, p. 57). Los conceptos ideales de la física parecen compartir con conceptos ideales de la economía, tales como "hombre económico" o "competencia perfecta de mercado", la característica de ser definibles con (eliminables en favor de) enun-

${ }^{16}$ En mi traducción: "Para que con estas palabras se signifique algo inequívoco, la sociología debe por su parte diseñar tipos ('ideales') 'puros' de estructuras del tipo que muestren en sí y de la mejor manera posible la unidad consecuente de una completa adecuación de sentido, pero justo por eso quizá tanto menos aparezcan en la realidad en esta forma absolutamente ideal y pura, al igual que una reacción física es calculada bajo la condición de un vacío absoluto." El original dice: "Damit mit diesen Worten etwas Eindeutiges gemeint sei, muß die Soziologie ihrerseits 'reine' ('Ideal')-Typen von Gebilden jener Arten entwerfen, welche je in sich die konsequente Einheit möglichst vollständiger Sinnadäquanz zeigen, eben deshalb aber in dieser absolut idealen reinen Form vielleicht ebensowenig je in der Realität auftreten wie eine physikalische Reaktion, die unter Voraussetzung eines absolut leeren Raums errechnet ist" (p. 560). La comparación con el caso del vacío es interesante, porque parece acercar los dos conceptos de tipo ideal.

${ }^{17}$ Puesto que sería difícil referirnos a todas las ciencias naturales, acotaremos el ámbito de este apartado a la física, considerada tradicionalmente la ciencia modelo entre todas las ciencias naturales; nuestros ejemplos serán tomados, por tanto, solamente de la física, pero en principio nuestra discusión podría ser aplicada a otras ciencias naturales.

Diánoia, vol. LII, no. 59 (noviembre 2007). 
ciados idealizacionales. ${ }^{18}$ Podemos concluir que son ideales en virtud de las idealizaciones que establecemos al definir el concepto. Los conceptos ideales no son, pues, diferentes e independientes de las idealizaciones, sino que se trata de la idealización misma revelada a nivel conceptual.

Podemos estar tentados a definir: " es un concepto ideal si las condiciones usadas en la caracterización de su significado son esencialmente contrafácticas." Al introducir la expresión "esencialmente" está claro que queremos evitar trivializaciones. Pero si aceptáramos esta definición, entonces debemos convenir en que los conceptos disposicionales (tales como soluble o frágil), que han sido analizados en términos de contrafácticos, caerían bajo la definición. En una primera instancia, la principal diferencia entre disposiciones y conceptos ideales parece ser que las disposiciones son propiedades reales en el sentido de que una adscripción disposicional implica enunciados de hecho (Mellor 1974, p. 173). Estos enunciados tienen una forma condicional, pero el antecedente es perfectamente posible o realizable. En el caso de los conceptos ideales, en cambio, las condiciones contrafácticas expresan una posibilidad meramente en principio; esto es así porque los conceptos ideales están construidos sobre la base de hipótesis que sólo pueden ser satisfechas de forma aproximada. Los conceptos ideales no tienen que ver con disposiciones, sino con conceptos límite. Nótese que la diferencia no es que sólo detrás de los conceptos ideales haya siempre una ley o enunciado legaliforme, puesto que las adscripciones disposicionales dependen usualmente de enunciados legaliformes: también hay una ley que involucra la estructura atómica o molecular de los cuerpos sólidos que explica por qué el cuerpo es soluble o frágil. Lo que ocurre verdaderamente es que, mientras en el caso de las disposiciones los enunciados contrafácticos no involucran idealizaciones (es decir, en ellos los antecedentes son realizables), en el caso de los conceptos ideales ocurre justamente lo contrario: conceptos ideales son aquellos que precisamente son descritos de manera esencial a través de idealizaciones.

${ }^{18}$ Barr (1971, pp. 259-260) observa correctamente que algunas idealizaciones podrían ser vistas igualmente como predicados. Tiene en mente idealizaciones que pueden ser analizadas como funciones de enunciados. Un ejemplo sería el siguiente: "todas las moléculas del gas $x$ son perfectamente elásticas y esféricas, poseen iguales masa y volumen, su tamaño es negligible y no ejercen fuerzas entre ellas excepto en las colisiones". Primeramente, este ejemplo parece ser el de un enunciado que surge de la suma de diferentes idealizaciones. Pero, desde el punto de vista lógico, no parece problemático tratarlo como una función de enunciado, que puede ser tratado a su vez como un predicado. 
Se dice que los conceptos ideales no tienen instancias. Nos enfrentamos, por tanto, al problema de justificar su uso en las teorías empíricas. Las generalizaciones con conceptos ideales como términos antecedentes o consecuentes son problemáticas, porque son infalsables o triviales. ${ }^{19}$ Si sustituimos los términos ideales ${ }^{20}$ por los enunciados idealizacionales que los definen y consideramos una generalización con una idealización como antecedente, tenemos un condicional vacuamente verdadero (porque su antecedente es siempre falso). De este modo debemos ofrecer un análisis de los conceptos ideales y de los enunciados idealizacionales de acuerdo con el que su uso pueda ser justificado de alguna manera. Ejemplos bien conocidos de conceptos ideales son "vacío perfecto", "gas ideal", "cristal perfecto", "cuerpo rígido" o "punto masa".

El carácter problemático de los conceptos ideales ha sido tratado de maneras diversas. Por ejemplo, de acuerdo con Nagel (1963), el uso de las generalizaciones que hacen referencia a tipos ideales está justificado sólo si son casos límite de un sistema más general de hipótesis que pueden ser empíricamente corroborados de una manera independiente. Esto es más o menos lo mismo que defendía Hempel (1965). Papineau (1976) sugiere entenderlos como la aplicación de la metodología lakatosiana de los programas de investigación. La aceptación de una generalización que se refiere a tipos ideales puede ser vista como un caso especial de adopción de un programa de investigación: esta particular clase de generalización desempeñaría el papel de la heurística negativa en la metodología de Lakatos; esto es, el núcleo duro del programa que los investigadores suponen irrefutable (por decisión metodológica). El papel de la heurística positiva, esto es, el de una guía del tratamiento de anomalías, sería ocupado por un conjunto de sugerencias sobre los límites en que las situaciones descritas por los tipos ideales satisfagan aproximadamente el término consecuente de la generalización de tipo ideal, sugerencias sobre cómo tales aproximaciones pueden ser diferen-

\footnotetext{
${ }^{19}$ Supogamos que hacemos una generalización con un concepto ideal en el antecedente. Como dice Papineau (1976, p. 137): "puesto que los tipos (o conceptos) ideales no tienen instancias, aceptar una generalización tal nunca nos permitirá inferir del hecho de que un estado de hechos se cumplió o se cumple, el hecho subsiguiente de que algo ha ocurrido u ocurrirá". Por otro lado, "las generalizaciones con tipos ideales como términos consecuentes no constituyen obviamente una buena explicación/predicción, puesto que las cosas que nos permitirían explicar/predecir nunca ocurren". La traducción es mía. Nótese que estamos hablando de las generalizaciones como proposiciones. Las proposiciones, como los enunciados, pueden tener también antecedentes y consecuentes.

${ }^{20}$ Términos ideales son los correlatos lingüísticos de los conceptos ideales.
} 
ciadas con respecto a algún grado o sobre qué generalizaciones pueden relacionar estas diferentes aproximaciones (Papineau 1976, p. 145).

Por consiguiente, mientras en el caso de Nagel y Hempel, la aceptación de una generalización de tipo ideal nos conmina a aceptar un sistema de generalizaciones independientemente corroborado al cual pertenecerían las generalizaciones de tipo ideal, en el caso de Papineau sólo se requiere que la generalización de tipo ideal nos ayude a construir un sistema tal, un sistema que sería construido gracias al trabajo conjunto de la heurística positiva y la negativa.

En una primera instancia, podemos convenir en que la metodología lakatosiana parece apropiada para capturar la idea del proceso idealización-concretización, puesto que, de acuerdo con Lakatos (1970), la heurística positiva nos permite elaborar un programa que consiste en una secuencia de modelos progresivamente más precisos y complejos de los fenómenos. Estos modelos son construidos tomando en cuenta progresivamente las complicaciones que habían sido negligidas en un principio. Ésta es una caracterización del proceso de concretización o de idealización. Lakatos da el ejemplo de la teoría de la gravedad: Newton elaboró su programa para un sistema planetario muy simplificado con un punto fijo representando al Sol y un único punto representando un planeta moviéndose alrededor del Sol. De este modelo derivó Newton la ley de la inversa del cuadrado. Pero, como este modelo contradice la tercera ley, Newton elaboró uno nuevo en el cual el Sol y el planeta se movían alrededor de un común centro de gravitación. Después consideró un modelo con varios planetas, pero como si sólo existiese la fuerza heliocéntrica. Finalmente, introdujo las fuerzas interplanetarias y consideró el Sol y los planetas no como puntos masa, sino con extensión. Con ello, Newton hizo más y más precisos sus modelos tomando en cuenta cada vez más factores.

Papineau tiene esencialmente dos argumentos contra la concepción defendida por Nagel y Hempel en relación con las generalizaciones de tipo ideal: en primer lugar, su concepción no justifica el uso de las generalizaciones de tipo ideal, pues éstas no pueden añadir nada al contenido empírico de los enunciados generales que relacionan las desviaciones de las condiciones ideales y los cambios que traen consigo. En segundo lugar, de aceptar tal concepción, tendríamos que rechazar muchas de las generalizaciones de tipo ideal de las ciencias sociales, ya que éstas no están usualmente integradas en un sistema de generalizaciones empíricamente confirmadas, y según Papineau esto sería una suerte de intolerancia metodológica. 
Debemos ofrecer una concepción de las generalizaciones de tipo ideal que justifique su uso y nos provea de una explicación de su contenido empírico, sin requerir que caigan necesariamente bajo el dominio de una teoría empírica confirmada independientemente. La solución va a tener que ver con el concepto de aproximación: lo interesante de los conceptos ideales es que estamos legitimados a aplicarlos a situaciones reales que satisfagan aproximadamente las condiciones involucradas en su caracterización. Para usar un concepto tal como vacío perfecto no necesitamos proporcionar una teoría empírica más general ya confirmada. Un buen ejemplo de esto sería el uso de Galileo del concepto de vacío antes de que fuera establecida una base empírica para este concepto en el siglo XVII gracias a las investigaciones de Boyle, Hooke y von Guericke. Como es bien conocido, Galileo tuvo que luchar contra la idea aristótelica de la imposibilidad física de un vacío. En ocasiones, es cierto, la ayuda de una teoría es necesaria para justificar el uso de teorías que se sabe son verdaderas o aplicables de modo aproximado o en condiciones muy ideales, aun cuando las viejas leyes sean consideradas todavía aplicables en suficiente número de casos. El carácter ideal o aproximadamente verdadero de muchas de estas teorías fue descubierto sólo después, una vez que se dispuso de teorías más precisas. Éste es el caso de la ley de la caída de los cuerpos de Galileo y la ley de la gravitación universal de Newton, que todavía se usan a pesar de saber que, estrictamente hablando, son falsas. Dejando a un lado las idealizaciones conscientemente efectuadas por Galileo (la eliminación, por ejemplo, de la resistencia del aire como factor distorsionador), el científico italiano creía haber establecido un descubrimiento de la constitución matemática del mundo y una definitiva verdad de la naturaleza.

Los conceptos ideales constituyen la base de las suposiciones que intentamos corroborar y, contra Papineau, las generalizaciones de tipo ideal son testables: si, después de muchas observaciones y experimentos, obtenemos como resultado que de tales generalizaciones no se puede decir que sean ni siquiera aproximadamente satisfechas, podemos rechazarlas. Los científicos usan las generalizaciones de tipo ideal para simplificar sus hipótesis: considerando el vacío perfecto, negligen la influencia de la resistencia del aire, cuya consideración complicaría las ecuaciones y, por tanto, los cálculos, además de las predicciones. Al mismo tiempo, tienen un valor heurístico: no pueden ser directamente testadas, pero pueden usarse como guía para la investigación y proporcionan una vía indirecta de test empírico basado en aproximaciones. Es aquí donde veo una solución posible al dilema presentado por Papineau (Papineau 1976, p. 141) y que según él representa un argumento con- 
tra la concepción aproximativa que acabamos de describir brevemente: Papineau cuestiona si está especificado qué grado de aproximación se requiere para que algo cuente como una instancia de un tipo ideal. Si no lo está, entonces las generalizaciones de tipo ideal son vacías, puesto que en tal caso sería arbitrario qué contaría como una instancia de tales generalizaciones. $\mathrm{Si}$, por el contrario, se hacen tales especificaciones y podemos trabajar dentro de un grado de aproximación preciso, entonces ¿de qué nos sirven las generalizaciones de tipo ideal? La solución al dilema consiste en recordar la noción de límite matemático. Las generalizaciones de tipo ideal nos muestran cuál es el objetivo al que deben tender nuestras investigaciones y nuestras predicciones. Si, conforme nos acercamos a un valor ideal, obtenemos resultados que confirman parcialmente nuestra idealización, estaremos cada vez más justificados en emplearla. Otra cuestión, de considerable importancia y relevancia para la ciencia actual, porque tiene que ver con fenómenos de turbulencia, por ejemplo, es el hecho de que muchas veces los límites en sí mismos fueran singulares; es decir, no dieran soluciones analíticas, pero en estos casos podemos postular o proponer soluciones convenientes para cada caso particular.

Un ejemplo de concepto ideal es el de gas ideal. Los gases ideales se definen como aquellos para los que el cociente $\frac{P V}{n T}$ es constante para todas las presiones. Pero los gases reales no son así; se aproximan a los ideales sólo a bajas presiones. Cuando la densidad del gas real disminuye y decrece asimismo la presión, el cociente $\frac{P V}{n T}$ tiende al mismo valor: $8,314 \mathrm{~J} / \mathrm{mol} \cdot \mathrm{K}$. Incluso el oxígeno, que se desvía mucho de los gases ideales a altas presiones, varía tan sólo un $1 \%$ de los gases ideales a presiones de entre 0 y 5 atmósferas. Los gases ideales se definen, pues, de acuerdo con una condición ideal, que no obstante puede ser aproximada en situaciones perfectamente determinables. No hay gases ideales, sino gases que en ciertas situaciones se comportan como si fueran gases ideales.

Un caso controvertido es el de cuerpo rígido. En sentido clásico, los cuerpos rígidos son definidos como aquellos en los cuales las distancias entre las partículas constituyentes permanece constante. Cuando aplicamos una fuerza a un cuerpo en un punto dado, se sigue de la definición y la mecánica clásica que esa fuerza debe ser transmitida de forma instantánea a todas las demás partes de ese cuerpo o, lo que es lo mismo, con velocidad infinita. Esto, sin embargo, es imposible si admitimos la teoría especial de la relatividad, pues la velocidad en la que es 
transmitida una fuerza no puede exceder la de la luz. En consecuencia, parece contradictorio que los científicos relativistas sigan trabajando con la definición clásica de cuerpo rígido. ${ }^{21}$

Algunos filósofos han sugerido que para los propósitos relativistas se use un concepto convenientemente modificado de cuerpo. Reichenbach (1958), por ejemplo, propuso definir los cuerpos rígidos de la siguiente manera: "Los cuerpos rígidos son sólidos que no están afectados por fuerzas diferenciales, o cuya influencia ha sido eliminada por correcciones; las fuerzas universales no se contemplan." 22 Las fuerzas universales son aquellas que afectan a todos los materiales de la misma forma y para las cuales no hay paredes aislantes. Todas las otras fuerzas son "diferenciales". Ésta es, por ejemplo, la noción de cuerpo rígido defendida por Nagel (1961). ${ }^{23}$ De acuerdo con su propuesta, un cuerpo es rígido si está libre de fuerzas perturbadoras y cuyas partes constituyentes permanecen invariantes en relación con sus distancias respectivas. Pero como Shapere $(1969$, p. 134) ha señalado, la noción relevante aquí no es ésta, de acuerdo con la cual prácticamente todos los cuerpos serían rígidos, sino la noción de cuerpo tal que, a pesar de la posible influencia de fuerzas externas, la distancia entre cualesquiera partes constituyentes permanece invariante.

Por tanto, la noción defendida por Reichenbach y Nagel no es satisfactoria. Los físicos han intentado una solución de conveniencia definiendo cuerpo rígido dentro del marco relativista. Así, Max Born propuso en 1910 la definición de cuerpos rígidos como aquellos para los que vale $\frac{\partial \delta s}{\partial t}=0$. Después se vio que la definición de Born tenía consecuencias inaceptables. Se probó, entre otras cosas, que un cuerpo que la satisfaga tendría sólo tres grados de libertad en lugar de los seis habituales en mecánica clásica. ${ }^{24}$

Existe, por supuesto, la posibilidad de argüir: (1) que el concepto clásico de cuerpo rígido es satisfecho en la realidad sólo aproximadamente, si bien las perturbaciones derivadas de la influencia de fuerzas externas es tan pequeño que podemos trabajar con él con fines prácticos, y (2) que la definición de cuerpo rígido puede ser modificada diciendo que son aquellos en los que los impulsos son transmitidos con la velocidad de la luz (Shapere 1969, pp. 136-138). Tenemos que la existencia

${ }^{21}$ Véase Anderson 1973, pp. 138 y 320.

${ }^{22}$ Reichenbach 1958, p. 22; la traducción es mía.

${ }^{23}$ Véase el cap. IX, sec. II, 1. Grünbaum (1963) es de esta misma opinión.

${ }^{24}$ Anderson 1973, p. 320. Para una discusión de la definición de Born, véase Pauli 1958, pp. 130-132.

Diánoia, vol. LII, no. 59 (noviembre 2007). 
de cuerpos rígidos en el sentido clásico es prohibida por la teoría de la relatividad. Tenemos también que la existencia de cuerpos rígidos en el sentido de la definición modificada (2) es meramente hipotética: la definición (2) tiene la consecuencia de que los cuerpos rígidos tienen una constante dieléctrica igual a 1 , aunque no exista ningún cuerpo con esa constante dieléctrica (se supone que es el valor de esa constante en el vacío). No obstante, esto parece ser una mera cuestión de hecho. No hay ninguna razón que podamos ofrecer desde la física relativista que prohíba la existencia de un cuerpo tal (Shapere 1969, p. 138). El uso del concepto de cuerpo rígido está justificado no importa qué definición escojamos, pues si elegimos (1), entonces los cuerpos rígidos se convierten en conceptos límite que pueden ser usados con fines prácticos, y si elegimos (2), efectuamos una hipótesis que es perfectamente compatible con la teoría de la relatividad y conveniente tanto a efectos teóricos como prácticos. De este modo, de acuerdo con la vía aproximativa, estamos legitimados en usar en cualquiera de los dos casos el concepto ideal de cuerpo rígido. Algo análogo sucedería con otros conceptos ideales usados en física.

\section{Vuelta a las ciencias sociales}

Acabamos de ver el uso y la función de los tipos ideales en la física, como ejemplo modelo de ciencia natural. Sin duda, lo dicho aquí no es inmediatamente extrapolable tout court a otras ciencias naturales donde los conceptos ideales desempeñan también un importante papel, como la biología. Sin embargo, nos atrevemos a conjeturar que un análisis cuidadoso revelaría más similitudes que diferencias. En este apartado vamos a volver a las ciencias sociales y a la cuestión de la demarcación, centrándonos en el caso de la economía, ya que tradicionalmente es considerada la ciencia humana más cercana a las ciencias naturales. Sin duda, un examen detenido de la función de los tipos ideales en ciencias sociales y de su relación con los tipos ideales e idealizaciones en ciencias naturales exigiría estudiar los casos de otras ciencias humanas, como la sociología o la historia, pero este examen, mucho más complejo y delicado, no puede ser abordado en el presente artículo. Se deja, pues, para una ocasión futura.

Muchos autores clásicos señalaron la importancia de las idealizaciones en economía: además de Marx, podemos citar a J.S. Mill y Carl Menger. Ambos se percataron de que hay muchas causas operantes en cualquier situación práctica y que no podemos dar una explicación cabal del fenómeno de investigación, es decir, los métodos convencionales 
de inducción no son suficientes y debemos emplear algún tipo de idealización. De esta forma, ambos fueron conducidos a una perspectiva a priori de la economía. ${ }^{25}$ De acuerdo con J.S. Mill, la inducción es claramente insuficiente, en virtud de la multiple y cambiante variedad de factores causales (que pueden actuar por combinación), pero al mismo tiempo reconoce que estos factores tienen sus causas particulares en una forma que es susceptible de investigación nomológica. ${ }^{26}$ Según Mill, las causas operantes en economía pueden, en líneas generales, ser cubiertas por leyes de la naturaleza humana. Para investigar las causas de fenómenos sociales particulares, se proponen hipótesis que hacen ciertas asunciones muchas veces ideales. Una suerte de proceso de idealización-concretización es admitido por Mill (1844) cuando dice:

si la asunción es correcta en la medida en que funciona y sólo difiere de la verdad en la misma forma que la parte difiere del todo, entonces las conclusiones que son correctamente deducidas de las asunciones constituyen una verdad abstracta; $\mathrm{y}$, cuando son completadas añadiendo o sustrayendo el efecto de las circunstancias no calculadas, son verdaderas en lo concreto y pueden ser aplicadas a la práctica. ${ }^{27}$

Es sintomático que, para Mill, podamos razonar aplicando este método a la política económica "con tanta certeza como en las partes más demostrativas de la física" (Mill 1844, p. 149).

Modernamente, la cuestión del papel de los tipos ideales en ciencias sociales se revitalizó y volvió a ser tema de discusión. Para empezar, fue inmediatamente rescatada por Schütz (1993) en el contexto de su

${ }^{25}$ Esto es más evidente en el caso de Menger: su teoría parece ser genuinamente a priori, como señala Cartwright (1994, p. 184). Mill define su método como deductivo en oposición a las críticas inductivistas de Ricardo, pero puede ser caracterizado mejor como una combinación de inducción y razonamiento deductivo. Véase también Cartwright 1989, pp. 170-171.

${ }^{26}$ De hecho, J.S. Mill prefiere hablar de "tendencias" y no de leyes causales con excepciones.

${ }^{27}$ Mill 1844, p. 149; la traducción es mía. Para un estudio de J.S. Mill, Marx y Friedman (y otros autores como John Ramsay McCulloch o Lionel Robbins) desde el punto de vista que nos ocupa, véase Hamminga y De Marchi 1994. Para una comparación entre J.S. Mill y Carl Menger, véase Cartwright 1994. Para la concepción de la idealización en Carl Menger, véase Birner 1994, pp. 279-281, que ve en Menger al genuino "Galileo de las ciencias sociales", en consabida oposición con Nowak, quien llamaba a Marx de ese modo. La contribución de Menger a la metodología se halla en su obra de 1883.

Diánoia, vol. LII, no. 59 (noviembre 2007). 
"construcción significativa del mundo social", dando lugar a una discusión que se prolongó gracias a la estela de influencia del teórico social alemán. Schütz no sólo rescató la cuestión, sino, más aún, puso de nuevo sobre la mesa el problema del sentido y la compresión del sentido, problemas que, como ya hemos dicho, no podemos tratar aquí. En el caso más concreto de la economía, debemos citar el caso de Milton Friedman. En su importante contribución a la metodología de la economía positiva, el premio Nobel de economía de 1976, muestra la importancia de los tipos ideales en economía teórica, refiriéndose a ejemplos tales como "perfecta competición" o empresas de monopolio. De acuerdo con Friedman, estos tipos ideales no se interpretan en un sentido descriptivo, sino "que se emplean para aislar los aspectos que son cruciales para un problema particular". ${ }^{28}$ Friedman compara los tipos ideales en economía con la idealización en física y pone el ejemplo de la ley de caída libre de Galileo. Como en el caso de la física, los teóricos de la economía no pueden testar la teoría a través de sus asunciones, sino que más bien son las asunciones, descriptivamente falsas (asunciones que proponen tratar ciertas entidades como si fueran tal y tal cosa, que sabemos no es el caso), las que debieran proporcionar un test indirecto de las hipótesis a través de sus implicaciones.

Siguiendo, pues, a Friedman (1984), podemos afirmar que el método idealizacional y el uso y función de los tipos ideales en economía no distan mucho de los de las ciencias naturales, como ya entrevimos al comentar los textos de Weber. Subsiste, eso sí, la cuestión de la demarcación, a juicio de Weber insalvable, entre ambas clases de ciencias.

Se ha insistido mucho en la comparación entre ciencias naturales y sociales intentando mostrar que no están tan separadas como inicialmente se podría pensar. Algunos de los insignes trabajos en este sentido son Kaufmann (1944), Gibson (1960) o Nagel (1961). Nos aventuramos a conjeturar que Weber no estaría necesariamente en desacuerdo con ellos, por lo menos en algunos puntos significativos. Lo que los trabajos de Weber intentaron mostrar es que, a pesar de esas similitudes, que es necesario señalar en un intento de análisis de la objetividad de las ciencias humanas como era el de Weber, hay elementos decisivos intrínsecos a la naturaleza, objetivos y metodología de las ciencias humanas que separan a éstas de las llamadas naturales. Esos elementos son, según Weber, la búsqueda de una comprensión del sentido de la acción social (como diferente de la explicación en ciencias naturales), la dificultad de aislar los múltiples y heterogéneos factores interferen-

${ }^{28}$ Friedman 1984, p. 233; la traducción es mía.

Diánoia, vol. LII, no. 59 (noviembre 2007). 
tes que tienen lugar en ciencias sociales (esto constituye también una diferencia con respecto a las ciencias naturales, pues en éstas esos factores interferentes se pueden clasificar y aislar mucho más fácilmente) y, finalmente, la cuestión valorativa en ciencias humanas, difícil de eliminar a pesar del postulado de neutralidad valorativa que el propio Weber demandaba al científico social. Por lo que hace a la naturaleza de las idealizaciones y la función de los tipos ideales, una de las principales diferencias señaladas en este artículo ha sido la multiplicidad, heterogeneidad, complejidad e intrincación de los factores interferentes en ciencias sociales, que dificultan tanto la formulación como la evaluación de las teorías en este tipo de ciencias. Es el conocido problema de la irracionalidad y la impredecibilidad en ciencias humanas. Sin embargo, se han sugerido al mismo tiempo algunas maneras de rescatar a las ciencias humanas de los elementos irracionales que volverían acientífico el discurso de estas disciplinas. La metodología weberiana, en especial la segunda noción de tipos ideales, que Weber aplica a la investigación sociológica en su obra de 1921, y el postulado de neutralidad valorativa, es indiscutiblemente un intento serio por solucionar estas dificultades. Uno de los puntos del presente artículo ha sido mostrar que, al introducir en la discusión la segunda noción de tipo ideal, Weber estaba en realidad acercando más de lo que quizá habría querido en 1904 las ciencias humanas a las naturales. Que las ciencias humanas son diferenciables de las ciencias naturales, tanto por sus objetivos como por su metodología, y además de modo prácticamente insalvable, es algo que Weber (1921) sigue sosteniendo, si bien, gracias a su segundo análisis, ambos tipos de ciencia estarían mucho más cercanas.

\section{BIBLIOGRAFÍA}

Aguilar Villanueva, Luis F., 1988 y 1989, Weber: La idea de la ciencia social, 2 vols., Coordinación de Humanidades-UnAM/Miguel Ángel Porrúa, México. Anderson, James L., 1973, Principles of Relativity Physics, Academic Press, Nueva York.

Barr, William F., 1971, "A Syntactic and Semantic Analysis of Idealization in Science", Philosophy of Science, vol. 38, no. 2, pp. 258-272.

Birner, Jack, 1994, "Idealizations and Theory Development in Economics. Some History and Logic of the Logic of Discovery", en Hamminga y de Marchi 1994, pp. 277-301.

Brown, Robert, 1963, Explanation in Social Sciences, Routledge and Kegan Paul, Londres.

Diánoia, vol. LII, no. 59 (noviembre 2007). 
Burger, Thomas, 1987, Max Weber's Theory of Concept Formation, Duke University Press, Durham, N.C.

Camardi, Giovanni, 2004, "Ideal Types and Scientific Theories", en F. Coniglione, R. Poli y R. Rollinger (comps.), Idealization XI: Historical Studies on Abstraction and Idealization, Poznan Studies in the Philosophy of the Sciences and the Humanities, Rodopi, Ámsterdam/Nueva York, vol. 82, pp. 273-285.

Cartwright, Nancy, 1994, "Mill and Menger: Ideal Elements and Stable Tendencies", en Hamminga y de Marchi 1994, pp. 171-188.

—_ 1989, Nature's Capacities and Their Measurement, Oxford University Press, Oxford.

Dray, William H., 1957, Laws and Explanation in History, Clarendon Press, Oxford.

Friedman, Milton, 1984, "The Methodology of Positive Economics", en Daniel M. Hausman (comp.), The Philosophy of Economics. An Anthology, Cambridge University Press, Cambridge, pp. 210-244 (1a. ed.: 1953).

Gibson, Quentin, 1960, The Logic of Social Enquiry, Routledge and Kegan Paul, Londres.

Grünbaum, Adolf, 1963, Philosophical Problems of Space and Time, Knopf, Nueva York.

Hamminga, Bert y Neil B. de Marchi (comps.), 1994, Idealization VI: Idealization in Economics, Rodopi, Ámsterdam.

Hempel, C.G., 1965, Aspects of Scientific Explanation and Other Essays in the Philosophy of Science, The Free Press, Nueva York; se cita según la versión en castellano: La explicación científica. Estudios sobre la filosofía de la ciencia, trad. Néstor Míguez y otros, Paidós, Buenos Aires, 1979.

Ibarra, Andoni y Thomas Mormann, 1998, "Datos, fenómenos y constructos teóricos - un enfoque representacional", trad. Víctor Sánchez de Zavala, Theoria, vol. 13/1, no. 31, pp. 61-87.

Kaufmann, Felix, 1944, The Methodology of the Social Sciences, Oxford University Press, Oxford.

Knight, Frank, 1921, Risk, Uncertainty and Profit, Houghton Mifflin Company, Boston/Nueva York.

Lakatos, Imre, 1970, "Falsification and the Methodology of Scientific Research Programmes", en The Methodology of Scientific Research Programmes, Cambridge University Press, Cambridge.

Laymon, Ronald, 1985, "Idealizations and the Testing of Theories by Experimentation", en Peter Achinstein y O. Hannaway (comps.), Experiment and Observation in Science, The MIT Press/Bradford Books, Boston, pp. 147173.

Mellor, D.H., 1974, "In Defense of Dispositions", The Philosophical Review, vol. 83, no. 2, pp. 157-181.

Menger, Carl, 1883, Untersuchungen über die Methode der Sozialwissenschaften, und der politischen Ökonomie insbesondere, Duncker und Humblot, Leipzig. 
Mill, John Stuart, 1844, "Essays on Some Unsettled Question of Political Economy", en The Collected Works of John Stuart Mill, ed. J.M. Robson, vol. 4, University of Toronto Press, Toronto, 1967.

Mises, Ludwig von, 1929/1976, Epistemological Problems of Economics, trad. George Reisman, New York University Press, Nueva York/Londres.

Mommsen, Wolfgang J., 1989, The Political and Social Theory of Max Weber: Collected Essays, The University of Chicago Press, Chicago.

Nagel, Ernest, 1963, "Assumptions in Economic Theory", American Economic Review, vol. suplementario 53/1, pp. 211-219.

_- 1961, The Structure of Science, Harcourt, Brace and World, Nueva York.

Nowak, Leszek, 1980, The Structure of Idealization: Towards a Systematic Interpretation of the Marxian Idea of Science, Reidel, Dordrecht.

Papineau, David, 1976, "Ideal Types and Empirical Theories", British Journal for the Philosophy of Science, vol. 27, pp. 137-146.

Pauli, Wolfgang, 1958, Theory of Relativity, Pergamon, Nueva York.

Ramsey, Jeffry L., 1994, "Ideal Reaction Types and the Reactions of Real Alloys", en PSA 1994, vol. 1, ed. David Hull, Micky Forbes y Richard M. Burian, PSA, East Lansing, Mich., pp. 149-159.

Reichenbach, Hans, 1958, The Philosophy of Space and Time, trad. Maria Reichenbach y John Freund, Dover/Nueva York. Originalmente publicado en alemán en 1928.

Rickert, H., 1986, Kulturwissenschaft und Naturwissenschaft, Reclam, Stuttgart; la obra original fue publicada en 1899. Se cita según la versión en castellano: Ciencia cultural y ciencia natural, trad. Manuel García-Morente, Espasa-Calpe, Buenos Aires, 1943.

Rudner, Richard S., 1966, Philosophy of Social Science, Prentice-Hall, Englewood Cliffs, N.J.

Shapere, Dudley, 1969, "Notes toward a Post-Positivistic Interpretation of Science", en Peter Achinstein y S. Barker (comps.), The Legacy of Logical Positivism, John Hopkins University Press, Baltimore, pp. 115-160.

Schelting, Alexander von, 1934, Max Weber Wissenschaftslehre. Das logische Problem der historischen Kulturerkenntnis. Die Grenzen der Soziologie des Wissens, J.C.B. Mohr (Paul Siebeck), Tubinga.

Schütz, Alfred, 1993, La construcción significativa del mundo social, trad. Eduardo J. Prieto, Paidós, Buenos Aires; 1a. ed. en alemán: 1932.

Suppe, Frederick, 1989, The Semantic Conception of Theories and Scientific Realism, University of Illinois Press, Urbana/Chicago.

Watkins, J.W.N., 1952, "Ideal Types and Historical Explanations", British Journal for the Philosophy of Science, vol. 3, pp. 22-43.

Weber, Max, 1988, Gesammelte Aufsätze zur Wissenschaftslehre, compilado por Johannes Winckelmann, J.C.B. Mohr (Paul Siebeck), Tubinga.

_ 1922, Wirtschaft und Gesellschaft, J.C.B. Mohr, Tubinga.

__ 1921, "Soziologische Grundbegriffe", en Weber 1988, pp. 541-581.

_ 1913 , "Über einige Kategorien der verstehenden Soziologie", en Weber 1988, pp. 427-474.

Diánoia, vol. LII, no. 59 (noviembre 2007). 
Weber, Max, 1904, “Die 'Objektivität' sozialwissenschaftlicher und sozialpolitischer Erkenntnis", en Weber 1988, pp. 146-214.

Wright, Georg Henrik von, 1979, Explicación y comprensión, trad. Luis Vega Reñón, Alianza Universidad, Madrid, cap. 4.

Recibido el 4 de abril de 2006; aceptado el 9 de noviembre de 2006. 